Proceedings

\title{
Enhancement of the Solubility of Rosuvastatin Calcium by Nanovesicular Formulation: A Systematic Study Based on a Quality by Design Approach ${ }^{\dagger}$
}

\author{
Marwa H. S. Dawoud 1,**, Ahmed M. Fayez ${ }^{2}$, Reem A. Mohamed ${ }^{2}$ and Nabila M. Sweed ${ }^{1}$ \\ 1 Department of Pharmaceutics, Faculty of Pharmacy, October University for Modern Sciences and Arts, \\ 6th of October 12451, Egypt; mdawoud@msa.eun.eg (M.H.S.D.), nsueed@msa.eun.eg (N.M.S.) \\ 2 Department of Pharmacology, Faculty of Pharmacy, October University for Modern Sciences and Arts, \\ 6th of October 12451, Egypt; afayez@msa.eun.eg (A.M.F.); ralia@msa.eun.eg (R.A.M) \\ * Correspondence: mdawoud@msa.eun.eg; Tel.: +20-1001-651-592 \\ + Presented at the 1st International Electronic Conference on Pharmaceutics, 1-15 December 2020; \\ Available online: https://iecp2020.sciforum.net/.
}

Citation: Dawoud, M.H.S.; Fayez, A.M.; Mohamed, R.A.; Sweed, N.M. Enhancement of the Solubility of Rosuvastatin Calcium by Nanovesicular Formulation: A Systematic Study Based on Quality by Design Approach. Proceedings 2021, 78, 34. https://doi.org/10.3390/

IECP2020-08698

Published: 1 December 2020

Publisher's Note: MDPI stays neutral with regard to jurisdictional claims in published maps and institutional affiliations.

Copyright: () 2020 by the authors. Licensee MDPI, Basel, Switzerland. This article is an open access article distributed under the terms and conditions of the Creative Commons Attribution (CC BY) license (http://creativecommons.org/licenses/by/4.0/).

\begin{abstract}
Rosuvastatin calcium (Rsv) is an effective statin, with a potent antihyperlipidemic effect. However, it suffers poor bioavailability owing to its poor solubility. Thus; encapsulating Rsv into a nanovesicular structure could overcome this problem. The aim of this work is to investigate the potential of solid lipid nanoparticles (SLNs) and nanostructured lipid carriers (NLCs) in enhancing the solubility of Rsv, using the quality by design (QbD) concept. A complete risk assessment study has been conducted, where the critical process parameters (CPPs), material attributes (MAs), and critical quality attributes have been identified using Ishikawa diagrams. Selected CPPs/MAs were screened and further upgraded to a $2^{4}$ full-factorial design to develop a design space with the optimized formula. The screened CPPs/MAs were tested on the particle size, the polydispersity index (PDI), the zeta potential ( $\zeta$-pot), and the entrapment efficiency (EE\%). A comprehensive approach for Rsv nanovesicular carriers has been conducted, where the NLCs showed better results than the SLNs. The optimized formula was prepared with 3\% total lipid content, $0.154 \%$ surfactant, and 9.4 $\mathrm{mg}$ drug. The optimized formula had a particle size of $310.5 \mathrm{~nm}$, with 0.243 PDI, a $\zeta$-pot of -24.7 $\mathrm{mV}$, and an $\mathrm{EE} \%$ of $93.87 \%$, and showed a sustained release of the drug for up to $72 \mathrm{~h}$. It successfully lowered total cholesterol, low density lipoprotein, and triglycerides, and elevated the levels of high density lipoprotein in rats, with better results as compared to the standard drug. Thus, a complete QbD study was conducted to explore experimental regions for many successful nanovesicular carriers for the enhancement of the solubility of poorly soluble drugs.
\end{abstract}

Keywords: quality by design; solid lipid nanoparticles; nanostructured lipid carriers; antihyperlipidemia

\section{Introduction}

Quality by design $(\mathrm{QbD})$ is a systematic science and risk-based approach that plays a great role in product and process understanding in order to achieve a safe product. The use of $\mathrm{QbD}$ in pharmaceutical formulation assures the quality of a pharmaceutical product through the use of scientific development and risk management tools, producing a high quality product in the most efficient manner [1]. Rosuvastatin calcium (Rsv) is one of the most effective statins, with the potential of reducing low-density lipoprotein (LDL), triglycerides (TGs), and increasing high-density lipoprotein (HDL). However, Rsv suffers from poor solubility and extensive first-pass effect, resulting in its poor bioavailability [2]. Solid lipid nanoparticles (SLNs) and nanostructured lipid carriers (NLCs) are vesicular nanoparticles made from physiologically accepted and biodegradable lipid fractions [3]. 
The objective of the current study is the application of a $\mathrm{QbD}$ approach in the optimization and formulation of Rsv; in an attempt to improve its solubility.

\section{Experiments}

\subsection{Materials}

Rosuvastatin calcium, A32700, was as a generous gift from the Global Napi Pharmaceutical Company (Cairo, Egypt), and Precirol ${ }^{\circledR}$ ATO 5 and Compritol ${ }^{\circledR} 888$ ATO were received as gifts from GatteFosse (Lyon, France). Tween ${ }^{\circledR} 20$ was purchased from Sigma (St. Louis, MO, USA) while Tween ${ }^{\circledast} 80$ from Scharlau (Barcelona, Spain). Stearic acid was obtained from Piochem (Giza, Egypt), Oleic acid from Oxford Labchem (Maharashtra, India), and Castor oil from UCCMA (Cairo, Egypt). Poloxamer ${ }^{\circledR} 188$ was from Caisson (Smithfield, UT, USA). All other chemicals and reagents were of analytical grade. Fructose for the in vivo study was obtained from UNIPHARMA Co. (El-Obour City, Cairo, Egypt), while sheep tail fat and hydrogenated oil were from obtained commercial sources. Sodium carboxymethyl cellulose was obtained from Chemajet Pharmaceutical Industries (Cairo, Egypt).

\subsection{Methods}

\subsubsection{Preparation of the Nanovesicular Carrier}

SLNs and NLCs were prepared by emulsification-ultrasonication method as reported by Das et al. [4].

\subsubsection{Characterization of Nanoparticles}

Particle size (PS), polydispersity index (PDI), and zeta potential ( $\zeta$-pot) measurements were performed using dynamic light scattering using a zetasizer after suitable dilution [5].The entrapment efficiency (EE\%) was measured indirectly by analyzing the free drug in the supernatant after centrifugation of the dispersion [5].

\subsubsection{Quality by Design Paradigm}

The quality target product profile (QTPP) of the current study is to enhance the solubility of Rsv. The average PS, PDI, $\zeta$-pot, EE\%, and drug release profile were taken as the influential critical quality attributes (CQAs) for the current study [6].

Ientification of the failure modes was performed using Ishikawa diagrams, to figure out the critical process parameters (CPPs) and material attributes (MAs) affecting the QTPP [7].

Screening of different solid lipids, liquid lipids, and surfactants for nanovesicular formulation: saturated solubility of the drug in different liquid lipids (oleic acid and castor oil) and SAA (Tween ${ }^{\circledR} 20$, Tween ${ }^{\circledR} 80$ and Poloxamer $^{\circledR}$ 188) was measured [8]. Compritol ${ }^{\circledR}$ 888 ATO, Precirol ${ }^{\circledR}$ ATO 5, and Stearic acid were tested for their ability to solubilize Rsv [9].

Optimization of Rsv-loaded SLN/NLC with the selected variables: a $2^{4}$ full factorial design was used for the optimization steps shown in Table 1. 
Table 1. The studied critical process parameters (CPPs)/ material attributes (Mas), their levels, and the composition of the 16 formulae as obtained from the factorial design, with the results of the critical quality attributes (CQAs).

\begin{tabular}{|c|c|c|c|c|c|c|c|c|}
\hline \multicolumn{5}{|c|}{ Factors } & \multicolumn{2}{|c|}{ Low Level (-1) } & \multicolumn{2}{|c|}{ High Level (+1) } \\
\hline \multicolumn{5}{|c|}{$X_{1}$ Lipid (\%) } & \multicolumn{2}{|r|}{1} & \multicolumn{2}{|c|}{3} \\
\hline \multicolumn{5}{|c|}{$X_{2}$ Surfactant (\%) } & \multicolumn{2}{|r|}{0.1} & \multicolumn{2}{|c|}{0.3} \\
\hline \multicolumn{5}{|c|}{$X_{3}$ Solid lipid (SL): Liquid lipid (LL) } & \multicolumn{2}{|r|}{$7: 3$} & \multicolumn{2}{|c|}{$10: 0$} \\
\hline \multicolumn{5}{|c|}{$X_{4}$ Drug amount (mg) } & \multicolumn{2}{|r|}{5} & \multicolumn{2}{|c|}{10} \\
\hline ن & $X_{1}$ & $X_{2}$ & $X_{3}$ & $\mathrm{X}_{4}$ & $\begin{array}{c}Y_{1}=\text { Particle Size } \\
(\mathrm{nm})\end{array}$ & $\begin{array}{c}\mathrm{Y} 2_{3}=\text { Polydispersity } \\
\text { index }\end{array}$ & $\begin{array}{c}Y_{3}=\text { Zeta potential } \\
(\mathrm{mV})\end{array}$ & $\begin{array}{c}Y_{4}=\text { Entrapment } \\
\text { efficiency }(\%)\end{array}$ \\
\hline F1 & -1 & -1 & 1 & -1 & $279.2 \pm 1.56$ & $0.452 \pm 0.05$ & $-14.3 \pm 2.07$ & $45.43 \pm 5.98$ \\
\hline F2 & 1 & -1 & -1 & -1 & $232.8 \pm 3.57$ & $0.175 \pm 0.09$ & $-19.2 \pm 1.87$ & $76.71 \pm 8.83$ \\
\hline F3 & 1 & 1 & 1 & -1 & $308.9 \pm 3.54$ & $0.589 \pm 0.13$ & $-14.1 \pm 4.08$ & $81.37 \pm 3.78$ \\
\hline F4 & -1 & 1 & 1 & -1 & $400.4 \pm 1.65$ & $0.643 \pm 0.08$ & $-12.9 \pm 2.98$ & $85.57 \pm 2.98$ \\
\hline F5 & 1 & -1 & 1 & 1 & $400.3 \pm 2.56$ & $0.384 \pm 0.04$ & $-14.7 \pm 0.98$ & $67.12 \pm 4.14$ \\
\hline F6 & 1 & -1 & -1 & 1 & $300.0 \pm 2.73$ & $0.228 \pm 0.05$ & $-21.4 \pm 3.09$ & $94.40 \pm 9.06$ \\
\hline F7 & 1 & 1 & -1 & 1 & $255.0 \pm 2.90$ & $0.292 \pm 0.09$ & $-16.6 \pm 4.09$ & $89.20 \pm 3.87$ \\
\hline F8 & -1 & 1 & -1 & 1 & $256.5 \pm 1.65$ & $0.400 \pm 0.04$ & $-16.3 \pm 1.87$ & $77.39 \pm 5.76$ \\
\hline F9 & -1 & -1 & -1 & 1 & $806.1 \pm 2.63$ & $0.538 \pm 0.06$ & $-18.1 \pm 3.04$ & $78.67 \pm 4.31$ \\
\hline F10 & 1 & 1 & 1 & 1 & $313.1 \pm 0.95$ & $0.270 \pm 0.08$ & $-13.5 \pm 1.98$ & $82.96 \pm 9.31$ \\
\hline F11 & -1 & -1 & -1 & -1 & $736.2 \pm 1.62$ & $0.479 \pm 0.02$ & $-11.8 \pm 3.50$ & $62.23 \pm 5.98$ \\
\hline F12 & 1 & -1 & 1 & -1 & $245.0 \pm 1.16$ & $0.237 \pm 0.04$ & $-10.3 \pm 2.05$ & $46.44 \pm 2.74$ \\
\hline F13 & 1 & 1 & -1 & -1 & $280.8 \pm 3.07$ & $0.262 \pm 0.05$ & $-12.3 \pm 2.08$ & $93.33 \pm 4.87$ \\
\hline F14 & -1 & 1 & 1 & 1 & $237.0 \pm 3.60$ & $0.445 \pm 0.07$ & $-11.1 \pm 1.95$ & $72.60 \pm 2.09$ \\
\hline F15 & -1 & 1 & -1 & -1 & $478.5 \pm 2.76$ & $0.815 \pm 0.04$ & $-11.0 \pm 1.08$ & $64.29 \pm 5.87$ \\
\hline F16 & -1 & -1 & 1 & 1 & $262.1 \pm 2.84$ & $0.348 \pm 0.04$ & $-10.9 \pm 1.10$ & $75.91 \pm 4.21$ \\
\hline
\end{tabular}

Data optimization and model validation: a design space was established based on the product desirability. An optimized formula $\left(\mathrm{O}_{1}\right)$ was prepared as suggested by the program and was evaluated, and compared with the expected results.

\subsubsection{In-Vitro Drug Release}

An in-vitro drug release study was tested using the dialysis membrane method in PBS at $\mathrm{pH}$ 7.4. The optimized formula was compared to the standard Rsv in distilled water (both containing $10 \mathrm{mg}$ Rsv), where the samples were withdrawn over a period of $72 \mathrm{~h}$ [10].

\subsubsection{In-Vivo Pharmacodynamics Study}

The in-vivo study was conducted on 24 male Wistar rats (170-200 g), which were allowed free access to water and food [11]. The rats were divided into two dietary groups. The normal-fat diet (NFD) group consisted of six rats fed on a NFD, and the high-fat diet (HFD) group consisted of 18 rats fed on a HFD. This diet regimen was continued for six weeks, and at week seven, the rats were fasted, anesthetized, and blood samples were withdrawn to measure triglycerides (TGs) and total cholesterol (TC) [12].

Animals were then grouped into four groups, the first group being the negative control rats, which were the NFD group and received plain sodium carboxymethyl cellulose aqueous solution. The second group was the hyperlipidemic positive control group, a HFD group in which the rats received plain sodium carboxymethyl cellulose aqueous solution, while the third group were a HFD group receiving Rsv in sodium carboxymethyl cellulose aqueous solution, and the last group were a HFD group receiving the optimized formula $\left(\mathrm{O}_{1}\right)$. After two weeks, animals were then anesthetized and fasting blood samples were taken to measure TGs, TC, high-density lipoprotein (HDL), and low-density lipoprotein (LDL) [12]. 


\section{Results and Discussion}

\subsection{Quality Target Product Profile and Risk Analysis}

Potential causes of each of the CQAs were outlined using Ishikawa diagrams, as represented in Figure 1.

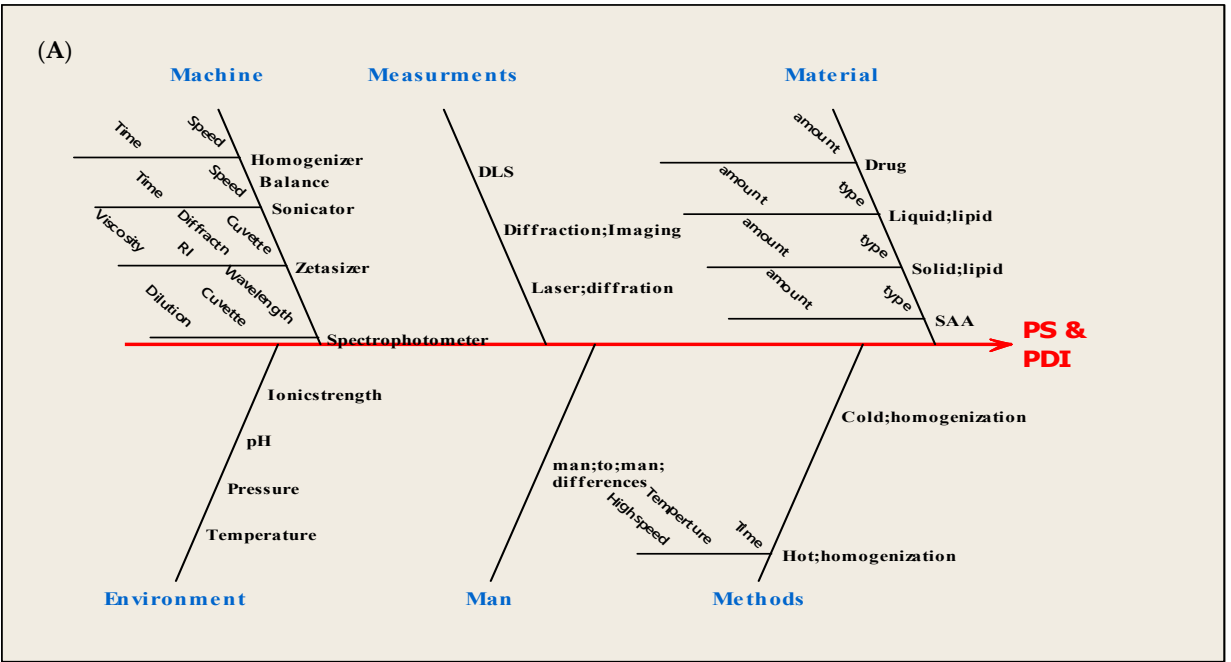

(B)

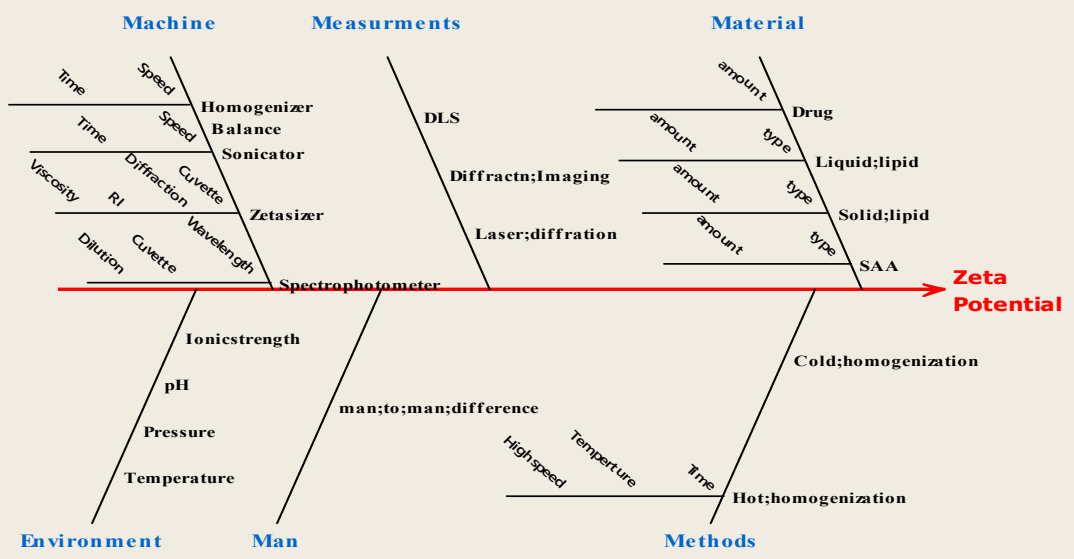

(C)

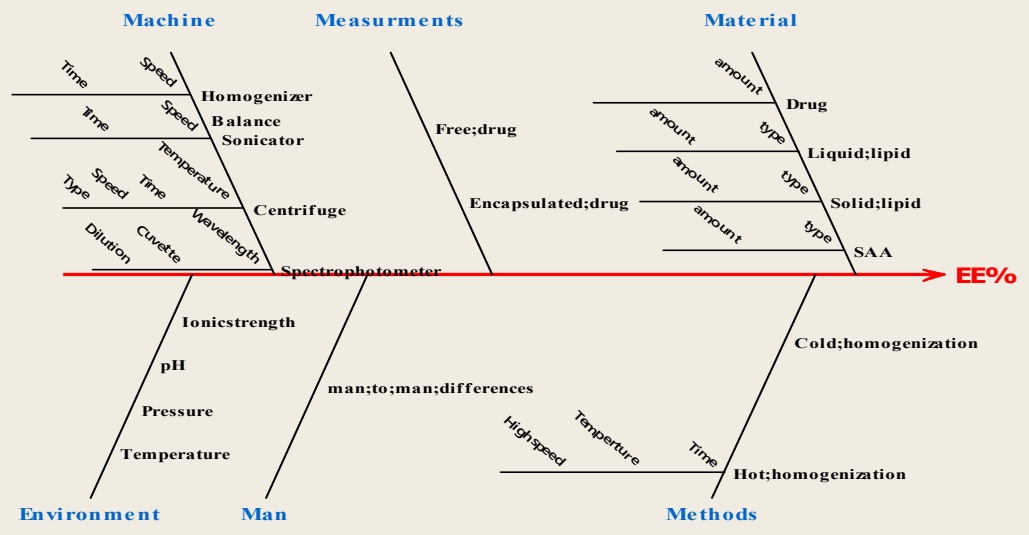

Figure 1. Ishikawa diagrams for different CQAs; (A) particle size (PS) and polydispersity index (PDI), (B) the zeta potential ( $\zeta$-pot), and (C) the entrapment efficiency (EE\%). 


\subsection{Screening of Different Solid Lipids, Liquid Lipids, and Surfactants (SAA) for Nanovesicular Formulation}

Rsv was found to be most soluble in Precirol ${ }^{\circledR}$ ATO 5 among the solid lipids, in oleic acid among the liquid lipids, and in Tween ${ }^{\circledR} 20$ among the SAA. Accordingly, these were the ingredients of choice for the nanovesicular preparations. The high solubility of Rsv in Precirol ${ }^{\circledR}$ ATO 5 may be due to the highly porous structure of Precirol ${ }^{\circledR}$ ATO 5 which allows more drug accommodation and solubility. The presence of methane sulfonamide hydrophilic moiety in Rsv resulted in the imparting of a slight hydrophilic nature, which in turn leads to its better solubility in Tween ${ }^{\circledR} 20$, than Tween ${ }^{\circledR} 80$ [13]. However, the hydrophobic nature of the drug allows Tween ${ }^{\circledR} 20$ to solubilize it more than Poloxamer $^{\circledR} 188$ [10].

\subsection{Response Surface Design Analysis}

Further analysis using ANOVA indicated that all models were significant, with the significant effect of CPPs/MAs on the measured CQAs at $(p<0.05)$.

\subsubsection{Particle Size Analysis}

The effect of the CPPs/MAs on the PS can be described as:

$P S=361.1-67.6 * X_{1}-44.7 * X_{2}-57.6 * X_{3}-6.2 * X_{4}+20.6 * X_{12}+82.7 * X_{13}+31.3 * X_{14}+55.9 * X_{23}-43.1 * X_{24}+7.6 * X_{34}$

An increase in $X_{1}$ or $X_{2}$ resulted in a significant reduction in the particle size. Probably, this may be due to the reduction in the surface tension when SAA\% increases, making the oil droplets smaller. In addition, SAA would be able to coat the oil droplets, which would stabilize the dispersion [4]. A larger particle size was observed when $X_{3}$ was decreased, i.e., the NLC was prepared, which may be attributed to the presence of the liquid lipid which might have increased the hydrodynamic diameter [14]. The effect of the drug amount was insignificant.

\subsubsection{PDI Analysis}

The effect of the CPPs/MAs on the PDI can be described as:

PDI $=+0.41-0.11 * X_{1}+0.051 * X_{2}+9.344 E-003 * X_{3}-0.048 * X_{4}+0.053 * X_{13}-0.067 * X_{24}$

A lower lipid content $\left(\mathrm{X}_{1}\right)$ resulted in a higher PDI value, which may be due to the insufficient amount of lipid to enclose Rsv, leading to the heterogenicity of the system [15]. The PDI was bigger at a high SAA\%, which could be attributed to the excess amount of SAA that may accumulate on the surface of the vesicles, resulting in an increase in the system's heterogenicity [8]. Moreover, the excess SAA might lower the surface tension to an extreme extent, which might rupture the vesicles and increase the system's heterogenicity [4]. A higher drug amount had a statistical significant lowering effect on the PDI, while the ratio between solid lipid and liquid lipid was insignificant.

\subsubsection{Zeta Potential Analysis}

The effect of the CPPs/MAs on the $\zeta$-pot can be described as:

$\zeta-$ pot $=+14-1.10 * \mathrm{X}_{1}+0.80 * \mathrm{X}_{2}+1.51 * \mathrm{X}_{3}-0.98 * \mathrm{X}_{4}-0.56 * \mathrm{X}_{13}+0.92 * \mathrm{X}_{23}-1.18 * \mathrm{X}_{34}$

The increase in the $\zeta$-pot by decreasing $X_{1}$ could be due to the reduction in the particle size by the increase in the total lipid content, which in turn reduces the surface area of the vesicles with less charge accommodation [16]. Moreover, the $\zeta$-pot was increased by the increase in the SAA\%, which could be due to molecular polarization and the adsorption of the surface acting agent in the water. The adsorbed SAA in the water could be absorbed to the emulsifier layer of the particle/water interface and form an electric double layer that is similar to an ionic state [17]. 


\subsubsection{EE\% Analysis}

The effect of the CPPs/MAs on the EE\% can be described as:

$\mathrm{EE} \%=76.46+4.33 * \mathrm{X}_{1}-6.25 * \mathrm{X}_{2}-5.02 * \mathrm{X}_{3}+5.38 * \mathrm{X}_{4}-4.49 * \mathrm{X}_{13}+4.61 * \mathrm{X}_{23}-5.63 * \mathrm{X}_{24}$

A significant increase in the EE\% was observed with the increase in the lipid content, which might be due to sufficient amounts of lipids that can be used to encapsulate the drug [15]. Moreover, a higher SAA\% resulted in a lower EE\%, which may be due to the disruption of the vesicles at a high SAA concentration [4]. When the NLC was prepared the EE\% increased, which could be due to the incorporation of a liquid lipid into a solid lipid, leading to a number of crystalline sequence disturbances and defects in the crystal lattice, which could in turn create space for the encapsulation of the Rsv molecules [10]. Finally, an increase in the EE\% was observed by the increase in the drug amount, which may be due to the increased availability of the Rsv, which consequently improves its retention within the vesicles [17].

\subsection{Model Validation, Data Optimization, and Control Strategy Establishment}

An optimized formula $\left(\mathrm{O}_{1}\right)$, with desirability 0.893 , was prepared (Table 2$)$. The validity of the design was established by comparing the observed results with the expected ones, which were found to statistically insignificant. A successful design space was established with a control space that ensures the reproducibility of the product.

Table 2.Composition of the optimized formula with the expected and the observed results.

\begin{tabular}{|c|c|c|}
\hline \multicolumn{2}{|c|}{ CPPs/MAs } & Level in Coded Value \\
\hline \multicolumn{2}{|c|}{ Total lipid content $\left(\mathrm{X}_{1}\right)$} & +1 \\
\hline \multicolumn{2}{|c|}{ SAA\% $\left(X_{2}\right)$} & -0.623 \\
\hline \multicolumn{2}{|c|}{ SL: $L L$ ratio $\left(X_{3}\right)$} & -1 \\
\hline \multicolumn{2}{|c|}{ Drug amount $\left(\mathrm{X}_{4}\right)$} & +0.992 \\
\hline \multirow{2}{*}{ CQA } & \multicolumn{2}{|r|}{ Results } \\
\hline & Expected & Observed \\
\hline PS (nm) & 352.345 & 310.5 \\
\hline PDI & 0.259 & 0.243 \\
\hline$\zeta$-pot $(\mathrm{mv})$ & -20.803 & -24.7 \\
\hline EE (\%) & 94.663 & 93.87 \\
\hline
\end{tabular}

\subsection{In-Vitro Drug Release Analysis}

The NLC optimized formula was able to release the drug in a sustained manner, as compared to the standard Rsv (Figure 2). The optimized formula was able to sustain the release for up to $72 \mathrm{~h}$. The drug on the surface of the vesicles resulted in an initial burst release which was followed by a sustained release pattern [18].

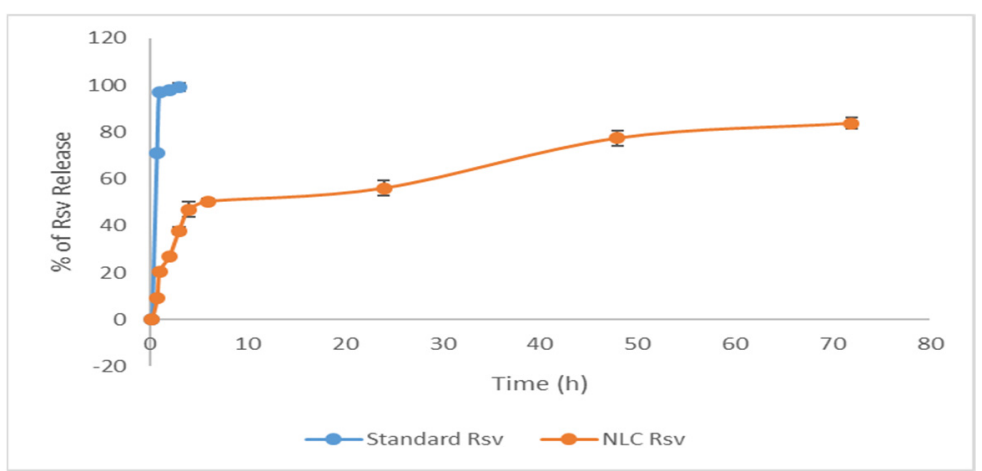

Figure 2. In-vitro release of Rsv from the optimized Rsv formula and standard Rsv. 


\subsection{In-Vivo Pharmacodynamics Study}

In agreement with previous reports [19], the hyperlipidemic positive control group exhibited a significant increase in TGs, TC, and LDL when compared to the negative control. Compared to standard Rsv, the optimized formula $\mathrm{O}_{1}$ significantly reduced serum TC level by $26.6 \%$ and LDL by $46 \%$. The elevation in HDL (13.95\%) and reduction in TGs (39\%) were not significant.

Statins have been reported to have side effects among quite a significant number of patients $(5-20 \%)$, with more side effects appearing at higher doses [20]. The use of the nanovesicular formulation of Rsv may increase its solubility, and hence its bioavailability. Moreover, the lipid formulation could induce bile secretion in the small intestine where the NLC would be associated with the bile salts, forming mixed micelles, and thus ensuring the NLC transition to the lymphatic circulation directly, bypassing the first-pass effect and promoting its better absorption [21]. All this resulted in improving and sustaining the antihyperlipidemic activity of Rsv NLC when compared to the standard Rsv.

\section{Conclusions}

The $\mathrm{QbD}$ approach was found to be very useful in formulation of a nanovesicular carrier loaded with Rsv. Several tools have been used in the risk assessment and design of experiments for the screening and the optimization of the nanovesicular carriers. A design space was established which defines the control strategy for the formulation of the Rsv-nanovesicular carrier. This control strategy gives the permitted ranges of the total lipid content, SAA\%, type of the nanovesicle, and the drug amount that produced the nano-vesicle with optimal PS, PDI, $\zeta$-potential, and EE\% for any further studies. The prepared optimized formula managed to significantly lower each of the TC, TGs, and LDL, and to elevate the HDL as compared to the positive control, thus proving the potential use of the Rsv-NLC as a successful antihyperlipidemic agent. A full and successful practical use of the QbD approach in pharmaceutical development was applied by the use of several advanced techniques, which could be considered as reliable reference for further nanovesicular carriers formulations.

Author Contributions: Conceptualization, M.H.S.D. and N.M.S.; methodology, M.H.S.D., N.M.S., A.M.F., and R.A.M; resources, M.H.S.D., N.M.S., A.M.F., and R.A.M; analysis, M.H.S.D., A.M.F., and R.A.M; data curation, M.H.S.D.; writing-original draft preparation, M.H.S.D., A.M.F., and R.A.M; writing-reviewing and editing, M.H.S.D. and N.M.S. All authors have read and agreed to the published version of the manuscript.

\section{Institutional Review Board Statement:}

The study was conducted according to the guidelines of the Declaration of Helsinki, and approved by the MSA University ethical committee, Egypt with approval number (PT15/EC15/2018F).

\section{Informed Consent Statement:}

This work contains no study involving humans.

Data Availability Statement:

The study didn't report any data.

Conflicts of Interest: The authors declare no conflicts of interest.

\section{References}

1. Xu, X.; Khan, M.A.; Burgess, D.J. A quality by design (QbD) case study on liposomes containing hydrophilic API: I. Formulation, processing design and risk assessment. Int. J. Pharm. 2011, 419, 52-59.

2. Kulkarni, N.; Ranpise, N.; Mohan, G. Development and Evaluation of Solid Self Nano-Emulsifying Formulation of Rosuvastatin Calcium for Improved Bioavailability. Trop. J. Pharm. Res. 2015, 14, 575.

3. Bahari, L.A.S.; Hamishehkar, H. The impact of variables on particle size of solid lipid nanoparticles and nanostructured lipid carriers; A comparative literature review. Adv. Pharm. Bull. 2016, 6, 143-151. 
4. Das, S.; Ng, W.K.; Tan, R.B.H. Are nanostructured lipid carriers (NLCs) better than solid lipid nanoparticles (SLNs): Development, characterizations and comparative evaluations of clotrimazole-loaded SLNs and NLCs?. Eur. J. Pharm. Sci. 2012, 47, 139-151.

5. Patil-Gadhe, A.; Pokharkar, V. Pulmonary targeting potential of rosuvastatin loaded nanostructured lipid carrier: Optimization by factorial design. Int. J. Pharm. 2016, 501, 199-210.

6. Xu, X.; Costa, A.P.; Khan, M.A.; Burgess, D.J. Application of quality by design to formulation and processing of protein liposomes. Int. J. Pharm. 2012, 434, 349-359.

7. Amasya, G.; Badilli, U.; Aksu, B.; Tarimci, N. Quality by design case study 1: Design of 5-fluorouracil loaded lipid nanoparticles by the W/O/W double emulsion-Solvent evaporation method. Eur. J. Pharm. Sci. 2016, 84, 92-102.

8. Basalious, E.B.; El-Sebaie, W.; El-Gazayerly, O. Application of Pharmaceutical QbD for Enhancement of the Solubility and Dissolution of a Class II BCS Drug using Polymeric Surfactants and Crystallization Inhibitors: Development of ControlledRelease Tablets. AAPS PharmSciTech 2011, 12, 799-810.

9. Singh, H.; Gupta, R.D.; Gautam, G. Formulation Development, Characterization, and in Vitro-in Vivo Study of Antihyperlipidemic Drug Rosuvastatin Calcium-Solid Lipid Nanoparticles. Asian J. Pharm. Clin. Res. 2018, 11, 436.

10. Patil-Gadhe, A.; Pokharkar, V. Montelukast-loaded nanostructured lipid carriers: Part i Oral bioavailability improvement. Eur. J. Pharm. Biopharm. 2014, 88, 160-168.

11. Mohamed, R.A.; Galal, O.; Mohammed, A.R.; El-Abhar, H.S. Tropisetron modulates peripheral and central serotonin/insulin levels via insulin and nuclear factor kappa B/receptor for advanced glycation end products signalling to regulate type-2 diabetes in rats. RSC Adv. 2018, 8, 11908-11920.

12. Schaalan, M.; El-Abhar, H.S.; Barakat, M.; El-Denshary, E.S. Westernized-like-diet-fed rats: Effect on glucose homeostasis, lipid profile, and adipocyte hormones and their modulation by rosiglitazone and glimepiride. J. Diabetes Complic. 2009, 23, $199-208$.

13. Sarfraz, R.M.; Ahmad, M.; Mahmood, A.; Minhas, M.U.; Yaqoob, A. Development and Evaluation of Rosuvastatin Calcium Based Microparticles for Solubility Enhancement: An In Vitro Study. Adv. Polym. Technol. 2017, 36, $433-441$.

14. Hu, X.; Zhang, Y.; Yang, J.; Wan, H. Influence of liquid lipid content on the properties of puerarin-loaded lipid nanoparticles. J. Chin. Adv. Mater. Soc. 2014, 2, 9-19.

15. Gokce, E.H.; Korkmaz, E.; Dellera, E.; Sandri, G.; Bonferoni, M.C.; Ozer, O. Resveratrol-loaded solid lipid nanoparticles versus nanostructured lipid carriers: Evaluation of antioxidant potential for dermal applications. Int. J. Nanomed. $2012,7,1841$.

16. Khames, A.; Khaleel, M.A.; El-Badawy, M.F.; El-Nezhawy, A.O.H. Natamycin solid lipid nanoparticles-sustained ocular delivery system of higher corneal penetration against deep fungal keratitis: Preparation and optimization. Int. J. Nanomed. 2019, 14, 2515-2531.

17. Wang, X. Development of antibody arrays for measuring biosimilar conformational comparability at molecular level. J. Bioequiv. Availab. 2012, 1, 24-26.

18. Sahu, A.K.; Kumar, T.; Jain, V. Formulation optimization of erythromycin solid lipid nanocarrier using response surface methodology. Biomed Res. Int. 2014, 2014, 689391.

19. Carmena, R. Type 2 diabetes, dyslipidemia, and vascular risk: Rationale and evidence for correcting the lipid imbalance. Am. Heart J. 2005, 150, 859-870.

20. Martin, P.D.; Warwick, M.J.; Dane, A.L.; Brindley, C.; Short, T. Absolute Oral Bioavailability of Rosuvastatin in Healthy White Adult Male Volunteers. Clin. Ther. 2003, 25, 2553-2563.

21. Khan, S.; Baboota, S.; Ali, J.; Khan, S.; Narang, R.S.; Narang, J.K. Nanostructured lipid carriers: An emerging platform for improving oral bioavailability of lipophilic drugs. Int. J. Pharm. Investig. 2015, 5, 182-191. 